\title{
Advances in turbulence measurements using new Correlation ECE and nT- phase diagnostics at ASDEX Upgrade
}

\author{
Simon J. Freethy ${ }^{1,2}$, Tobias Görler ${ }^{1}$, Alex J. Creely ${ }^{2}$, Garrard D. Conway ${ }^{1}$, Severin S. Denk ${ }^{1,3}$, Tim Happel $^{1}$, Pascale \\ Henniquin $^{4}$, Christian Koenen ${ }^{5}$, Anne E. White ${ }^{2}$, and The ASDEX Upgrade team ${ }^{1}$ \\ ${ }^{1}$ Max Plank Institute for Plasma Physics, 85748 Garching, Germany \\ ${ }^{2}$ Plasma Science and Fusion Center, Massachusetts Institute of Technology, Cambridge, MA 02139, USA \\ ${ }^{3}$ Physik-Department E28, Technische Universität München, 85748 Garching, Germany \\ ${ }^{4}$ Laboratoire de Physique des Plasmas, Ecole Polytechnique, 91128, Palaiseau cedex, France \\ ${ }^{5}$ Lehrstuhl für Hochfrequenztechnik, Technische Universität München, Arcisstr. 21, 80333 München
}

\begin{abstract}
Guided by predictions from nonlinear gyrokinetic simulations, two new turbulence diagnostics were designed and installed at ASDEX Upgrade (AUG) to probe the fundamentals of ion-scale turbulent electron heat transport. The first, a 30-channel correlation ECE (CECE) radiometer (105-128 GHz, 2nd harmonic X-mode), introduces a novel channel comb arrangement. This allows measurements of high radial resolution profiles $(0.5<r / a<0.8)$ of low- $k\left(k_{\theta} \rho_{s}<0.3\right)$ temperature fluctuation amplitudes, frequency spectra and radial correlation length profiles in unprecedented detail. The second diagnostic is formed by the addition of two W-band and one V-band X-mode reflectometers on the same line of sight as the CECE to enable measurements of the phase angle between turbulent density and temperature fluctuations. Historically, the radial alignment between reflectometer and radiometer has been a challenge due to the requirement that alignment is achieved within a radial correlation length $(<5-10 \mathrm{~mm})$. This challenge is significantly alleviated by using the CECE channel comb arrangement and the maximal coherence between reflectometer and radiometer can be unambiguously captured. Measurements of these quantities have been made in an AUG L-mode plasma, at the same radial location and have provided simultaneous quantitative constraints on realistic gyrokinetic simulations [Physics of Plasmas 25, 055903 (2018)] using the gyrokinetic code GENE. Here we present diagnostic detail for this study.
\end{abstract}

\section{Introduction}

Understanding the turbulent driven heat flux in a tokamak remains one of the key goals of fusion research. Anomalous transport up to two orders of magnitude above what one would expect from neoclassical theory is observed and this is now understood to be caused by turbulent fluctuations in the plasma density, temperature and potential, originating from drift-wave like instabilities which grow and non-linearly saturate $[1,2]$. In order to study in detail the turbulence giving rise to electron heat transport, the ASDEX Upgrade (AUG) correlation ECE (CECE) diagnostic was significantly upgraded, introducing a channel comb arrangement. This new diagnostic then measures high radial resolution fluctuation amplitude, $\delta T_{e \perp} / T_{e}$ profiles. The AUG CECE channel comb also provides the first measurements of high radial resolution profiles of $L_{r}\left(T_{e \perp}\right)$ using the CECE channel comb, showing the proportionality of $L_{r}\left(T_{e \perp}\right)$ to the ion sound gyroradius, $\rho_{s}$. Measurements of the radial correlation length of the temperature perturbations, $L_{r}\left(T_{e \perp}\right)$, have been reported previously $[3,4]$, but are nonetheless rare and the relationship to $\rho_{s}$ never explicitly demonstrated.

The heat flux driven by turbulent fluctuations depends not only on the amplitudes of the fluctuating quantities, but on their relative coherencies and phase angles [5]. Experimental measurements of these cross-field quantities are rare due to the difficulty in attaining them, but offer extremely valuable information with which to constrain high fidelity models of turbulence. For example, the phase angle between temperature and density fluctuations $\alpha_{n T}$ has been measured in the core plasma by the combination of a reflectometer and radiometer [5, 6]. We follow this approach here, combining two W-band $(75-110 \mathrm{GHz})$ and one $\mathrm{V}$ band $(50-75 \mathrm{GHz}) \mathrm{X}$-mode reflectometers along the same line of sight as the AUG CECE. We find that the CECE channel comb significantly improves the reliability of the radial alignment between radiometer and reflectometer. All quantities have been measured simultaneously at the same radius in dominantly electron heated L-mode plasma and have been compared directly to nonlinear ion scale GK simulations [7]. It is found that both electron and ion heat flux can be matched, along with $\alpha_{n T}$ and $L_{r}\left(T_{e \perp}\right)$, however $\delta T_{e \perp} / T_{e}$ is higher in the simulations than measured.

\section{Correlation ECE diagnostic}

In order to investigate the ion-scale electron heat transport at ASDEX Upgrade, a Correlation ECE receiver was 


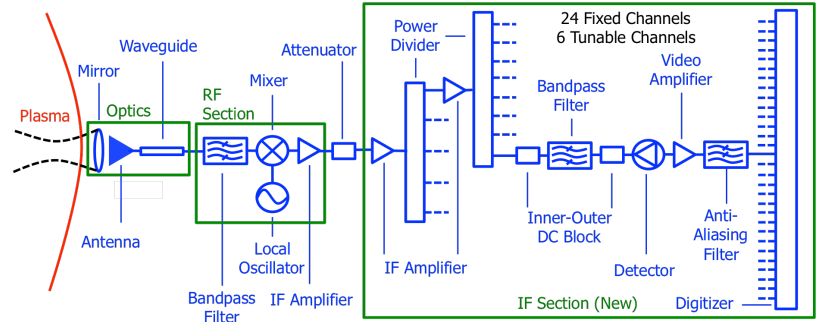

Figure 1. Reproduced from [8]. Block diagram showing the layout of the new AUG CECE diagnostic.

designed and built which employs both a high channel number and a novel channel comb arrangement. Three interchangable radio frequency (RF) sections employ tunable oscillators in the range $105.5-109.1 \mathrm{GHz}, 110.2-113.8$ $\mathrm{GHz}$ and $113.5-117 \mathrm{GHz}$, which in turn feed a filter bank of 24 fixed channels covering the range $4-8 \mathrm{GHz}$ in steps of either 100 or $200 \mathrm{MHz}$, as shown in Figure 1. This results in a radial spacing on the order of the ECE linewidth ensuring maximal spatial resolution of between $2-4 \mathrm{~mm}$. Four additional tunable YiG filters increase the frequency range up to $14 \mathrm{GHz}$. This configuration was guided by ECE modelling using a radiation transport model [9] and existing non-linear gyrokinetic (GK) simulations for predictions of the radial correlation length of the perpendicular electron temperature fluctuations $L_{r}\left(T_{e \perp}\right)$.
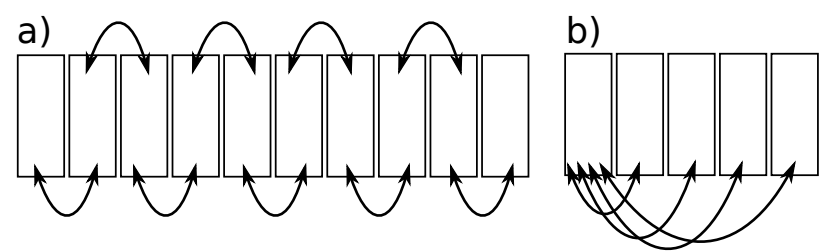

Figure 2. Schematic showing example channel combinations for high radial resolution $\delta T_{e} / T_{e}$ profiles (a) and correlation length measurements (b). Reproduced from [7].

The novel channel comb allows the measurement of high resolution $\delta T_{e \perp} / T_{e}$ and $L_{r}\left(T_{e \perp}\right)$ profiles. $\delta T_{e \perp} / T_{e}$ is calculated from a nearest neighbour cross-correlation as shown in Figure 2 (a). The correlation length is calculated from cross-correlations of increasing distance as shown in Figure 2 (b). In this case, the reference channel can be changed and the process repeated, allowing a correlation length profile to be built up. Figure 3 shows $\delta T_{e \perp} / T_{e}$ and $L_{r}\left(T_{e \perp}\right)$ profiles for a medium density L-mode plasma with $T_{e}>T_{i}$. It has previously been reported for density fluctuations that $L_{r}\left(n_{e}\right) \simeq 5-10 \rho_{s}[10]$. We can use this data to confirm for the first time a similar trend for the electron temperature fluctuations. In our case $L_{r}\left(T_{e \perp}\right)=8.5 \rho_{s}$, as shown in Figure 3 (b).

Non linear gyrokinetic results for these quantities at $\rho_{\text {pol }}=0.86$ produced using the GENE gyrokinetic code [11] and described in a previous publication [7] are also shown in the figure, denoted by the black stars. As you can see, reasonable agreement between GENE and the ex-
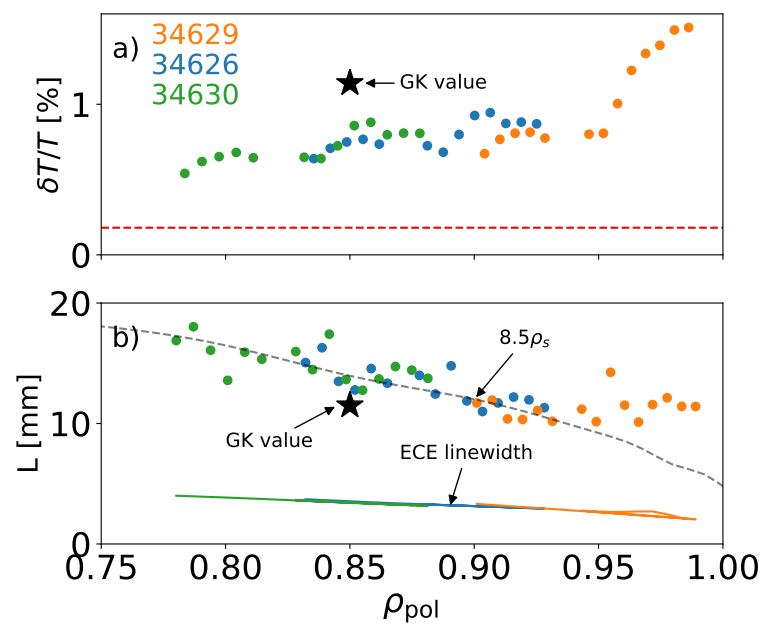

Figure 3. High radial resolution $\delta T_{e, \perp} / T_{e}$ profile (a) and correlation length profile, $L_{r}\left(T_{e \perp}\right)$, (b) as measured by the AUG CECE diagnostic. $L_{r}\left(T_{e \perp}\right)$ is shown to agree well with $8.5 \rho_{s}$ in this case, with a jump at $\rho_{\mathrm{pol}}=0.95$. The ECE linewidth $w_{\mathrm{ECE}}$ and gyrokinetic values are also shown for comparison.

periment is found, although the code slightly over predicts $\delta T_{e, \perp} / T_{e}$.

\section{$3 \mathrm{nT}$ cross-phase diagnostic}

The cross-phase angles between the fluctuating fields are important quantities for the validation of gyrokinetic models, since they determine the magnitude of the heat flux along with the fluctuation amplitudes. The most experimentally accessible quantity for core fluctuations is the phase angle between temperature and density fluctuations. This quantity has been shown to vary when experimentally varying the $T_{e}$ normalised gradient scale length $1 / L_{T e}$, which is a drive for the trapped electron (TEM) and electon temperature gradient (ETG) modes [12]. This work indicates that $\alpha_{n T}$ is an experimental measure of the balance of TEM and ITG structure present in the turbulence. $\alpha_{n T}$ has been measured previously by using a combination of a reflectometer for the density fluctuations and a radiometer for the temperature fluctuations, which share the same line of sight to the plasma $[5,6]$ and a similar approach is taken here. As can be seen from Figure 4, the CECE is installed on the same oversized waveguide as $\mathrm{W}$ and $\mathrm{V}$ band X-mode reflectometers. They are coupled via a $3 \mathrm{~dB}$ wire-grid splitter and share the antenna and focussing mirror, giving both diagnostics the same line of sight. Then, if radial alignment can be achieved by choosing the reflectometer frequency appropriately, cross-correlation of radiometer and reflectometer signals may be used to calculate $\alpha_{n T}$.

A significant problem in the operation of a combination reflectometer radiometer $\alpha_{n T}$ diagnostic is the stringent radial alignment constraint. The frequency of the reflectometer should be chosen with sufficient accuracy such 


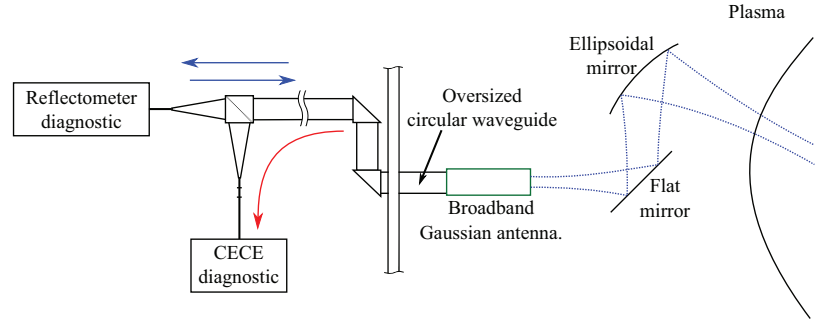

Figure 4. Adapted from [13]. A schematic of the CECE waveguide and optical setup. The CECE is set up to use the same line of sight as a reflectometer, coupled via a $3 \mathrm{~dB}$ wire-grid splitter.

that the cut-off position aligns well within a turbulent radial correlation length of an ECE radiometer channel, i.e. $<5 \mathrm{~mm}$. Within the uncertainties of the experiment, the chances of aligning a single reflectometer and radiometer channel first time are small. Figure 5 shows the $\mathrm{X}$ mode cut-off and cyclotron harmonics for the target Lmode plasma. One can see that a $20 \%$ uncertainty in the density gives rise to a significant uncertainty in the cutoff position. One way of alleviating this issue is to use the CECE channel comb to continuously cover a larger radial range, and one can easily estimate that a $5 \mathrm{GHz}$ comb would be required in this case. This is sufficient to achieve radial alignment using only a single plasma discharge, as we may also take advantage of 3 simultaneous reflectometer frequencies, thus significantly improving the reliability, productivity and usefulness of the diagnostic.

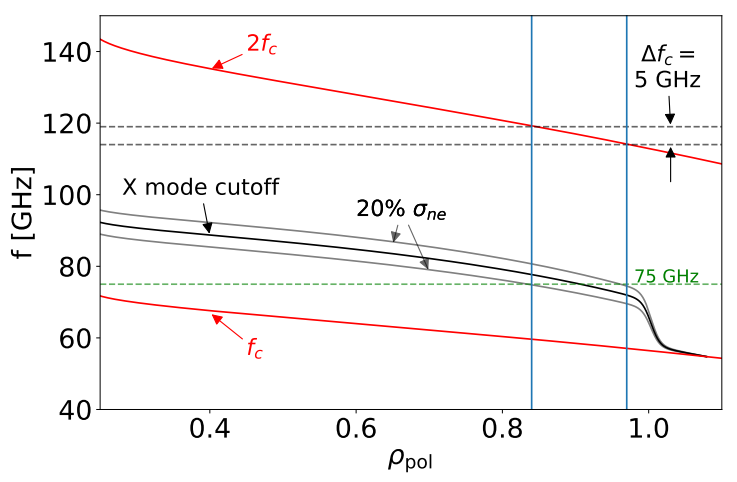

Figure 5. The first two cyclotron harmonics and the X-mode cutoff frequency profile. A $20 \%$ error in the density profile leads to a significant uncertainty in the radial position of the reflectometer. For this case, a CECE channel comb with a bandwidth of $5 \mathrm{GHz}$ should be sufficient to capture the coherence between reflectometer and radiometer.

The chosen technique is to illuminate the plasma at a fixed frequency, and the resulting amplitude fluctuations in the return waves are then correlated with each of the CECE channels in turn. It is then possible to build up a profile of the coherence between reflectometer and radiometer (refl.ECE) as shown in Figure 6 (a) and (b). These panels show the coherence averaged between $0-100 \mathrm{kHz}$ for refl.-ECE (black squares) and between radiometer channels (ECE-
ECE, orange triangles) with respect to a reference channel chosen to line up with the peak refl.-ECE coherence. The width of the latter is dominated by the $L_{r}\left(T_{e \perp}\right)$ as shown in section 2. One can see that the refl.-ECE coherence function is asymmetric, being broader towards higher radii compared to the ECE-ECE coherence function. This suggests that the reflectometer scattering carries information about density fluctuations away from the cut-off location and is thus correlated with fluctuations more than a radial correlation length away from the cut-off.

Figure 6 (c) and (d) show the cross-phase averaged from $10-40 \mathrm{kHz}$ for refl.-ECE correlations (black squares), ECE-ECE correlations (orange triangles). One can see that there is a slope in the average cross-phase present both in ECE-ECE and refl.-ECE correlations and this is due to a finite radial time delay of the turbulent structures. This is considered to originate from the finite turbulent eddy tilt angle, as there is no poloidal projection to this line of sight. Both refl.-ECE and ECE-ECE correlations show approximately the same phase behaviour with radius.

The advantage of the channel comb is then clear in the presence of such a behaviour in the cross-phase, as the ECE-ECE correlations are required to correct the phase trend in the refl.-ECE correlations for a robust measurement. Further, the measurement is only valid at peak coherence and without knowledge of the linear trend in the phase, the tolerance on radial alignment between ECE and reflectometer position is only a small fraction of the radial correlation length $\left(< \pm 0.4 L_{r}\right.$ or $\left.< \pm 2-4 \mathrm{~mm}\right)$, despite the fact that the reflectometer is sensitive to fluctuations over a broader radial range than the ECE. This degree of alignment can only be achieved with a channel comb in either ECE or reflectometer, or by painstakingly repeating the measurement with minute adjustments to achieve the same result.

\section{Conclusions}

A new Correlation ECE (CECE) and density temperature phase angle, $\alpha_{n T}$, diagnostic has been designed and built for ASDEX upgrade. The system uses 24 non-overlapping frequency channels arranged in a comb pattern, which when the finite channel bandwidth, Doppler broadening, relativistic broadening and radiation transport are considered, give near continuous radial coverage of a small radial region. This diagnostic gives detailed measurements of the radial correlation length of the temperature fluctuations $L_{r}\left(T_{e \perp}\right)$ and confirms for the first time the proportionality of $L_{r}\left(T_{e \perp}\right)$ to the local thermal ion gyroradius $\rho_{s}$. The addition of $2 \mathrm{~W}$-band and $1 \mathrm{~V}$-band $\mathrm{X}$-mode reflectometers along the same line of sight allows the measurement of the phase angle between density and temperature fluctuations $\alpha_{n T}$. The channel comb significantly improves the reliability of the diagnostic, and it has been shown that the condition of radial alignment can be more stringent than previously thought due to a linear trend in the cross-phase as a function of the separation between reflectometer cut-off and ECE channel position. High fidelity, ion-scale, non-linear gyrokinetic simulations performed agree well with the experimentally inferred electron and 

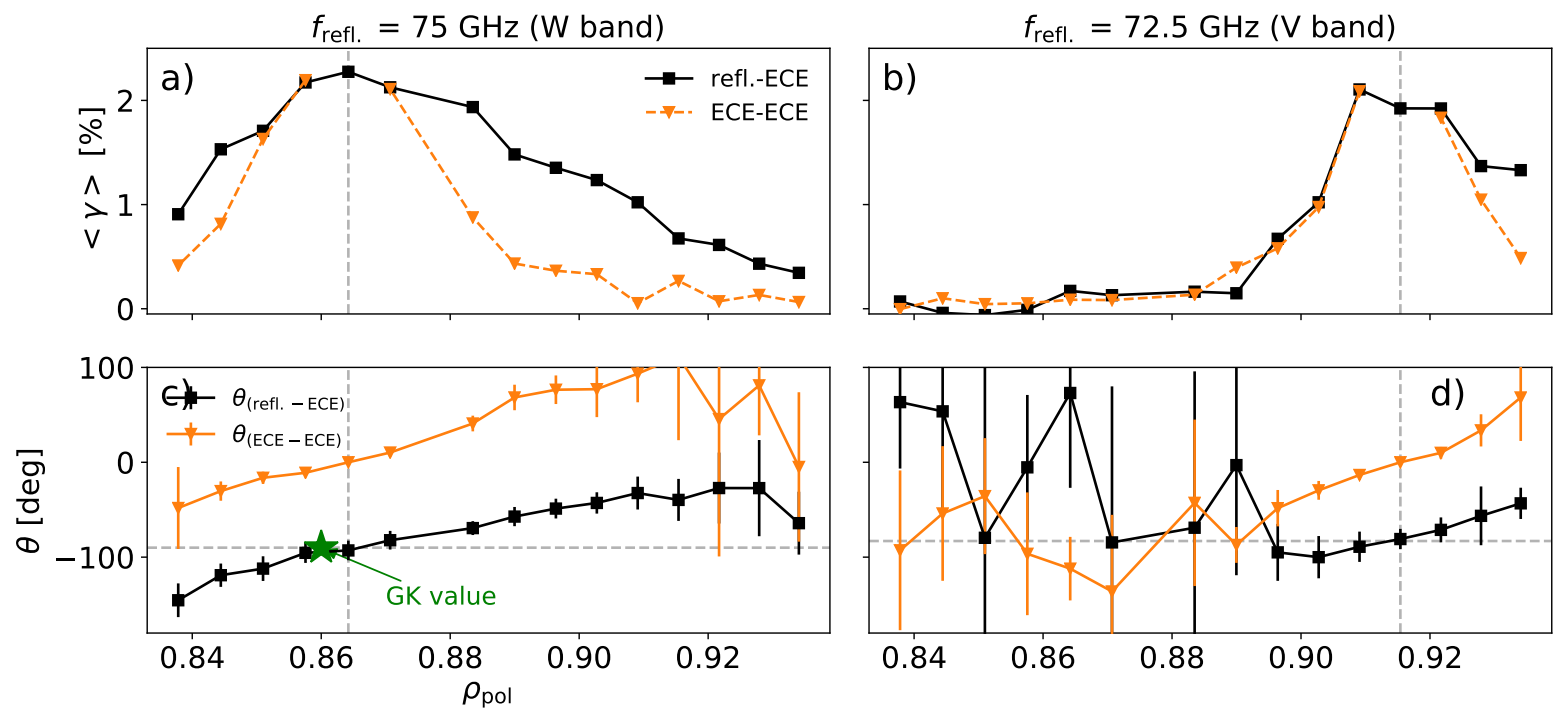

Figure 6. (a) and (b): Coherence between ECE channels (orange triangles) and ECE and reflectometer amplitude (black squares) averaged between $0-100 \mathrm{kHz}$ for reflectometer probing frequency of $75 \mathrm{GHz}$ and $72.5 \mathrm{GHz}$. The ECE coherence is scaled by 0.4 . (c) and (d): The cross phases averaged between 10-40 kHz between the ECE channels (orange triangles), ECE and reflectometer amplitude (black squares).

ion heat fluxes, while simultaneously matching the measured $\alpha_{n T}$ and $L_{r}\left(T_{e \perp}\right)$. Temperature fluctuation amplitudes are slightly above those measured in the plasma.

\section{Acknowledgements}

This work is supported by the US DOE under grants DESC0006419 and DE-SC0017381, and was performed in the framework of the Helmholtz Virtual Institute on Plasma Dynamical Processes and Turbulence Studies using Advanced Microwave Diagnostics. It has also been carried out within the framework of the EUROfusion Consortium and has received funding from the Euratom research and training programme 2014-2018 under grant agreement No 633053. The views and opinions expressed herein do not necessarily reflect those of the European Commission. The gyrokinetic simulations have been performed at the MARCONI supercomputer at CINECA and the MPCDF supercomputing facilities.

\section{References}

[1] J. Connor, H. Wilson, Plasma Physics and Controlled Fusion 36, 719 (1994)

[2] W. Horton, Reviews of Modern Physics 71, 735 (1999)

[3] L. Porte, S. Coda, T. Goodman, A. Pochelon, V. Udintsev, V. Vuille, EPJ Web of Conferences 32, 03007 (2012)

[4] M. Fontana, L. Porte, S. Coda, O. Sauter, Nuclear Fusion 58, 024002 (2018)
[5] A. White, W. Peebles, T. Rhodes, C. Holland, G. Wang, L. Schmitz, T. Carter, J. Hillesheim, E. Doyle, L. Zeng et al., Physics of Plasmas 17, 056103 (2010)

[6] M. Häse, M. Hirsch, H.J. Hartfuss, Review of Scientific Instruments 70, 1014 (1999)

[7] S.J. Freethy, T. Görler, A.J. Creely, G.D. Conway, S.S. Denk, T. Happel, C. Koenen, P. Hennequin, A.E. White, Physics of Plasmas 25, 055903 (2018)

[8] A.J. Creely, S.J. Freethy, W.M. Burke, G.D. Conway, R. Leccacorvi, W.C. Parkin, D.R. Terry, A.E. White, Review of Scientific Instruments 89, 053503 (2018)

[9] S. Denk, R. Fischer, O. Maj, E. Poli, J. Stober, U. Stroth, B. Vanovac, W. Suttrop, M. Willensdorfer, EPJ Web of Conferences 147, 02002 (2017)

[10] T.L. Rhodes, J.N. Leboeuf, R.D. Sydora, R. Groebner, E.J. Doyle, G.R. McKee, W.A. Peebles, C.L. Rettig, L. Zeng, G. Wang, Phys. Plasmas 9, 2141 (2002)

[11] F. Jenko, W. Dorland, Plasma Physics and Controlled Fusion 43, A141 (2001)

[12] J. Hillesheim, J.. DeBoo, W. Peebles, T. Carter, G. Wang, T. Rhodes, L. Schmitz, G. McKee, Z. Yan, G. Staebler et al., Physics of Plasmas 20, 056115 (2013)

[13] S.J. Freethy, G.D. Conway, I. Classen, A.J. Creely, T. Happel, A. Köhn, B. Vanovac, A.E. White, Review of Scientific Instruments 87, 11E102 (2016) 